\title{
ANÁLISE DOS PARÂMETROS FÍSICOS E BIOQUÍMICOS DE PACIENTES COM INSUFICIÊNCIA RENAL CRÔNICA EM INÍCIO DE TERAPIA RENAL HEMODIALÍTICA
}

\author{
ANALYSIS OF THE PHYSICAL AND BIOCHEMICAL PARAMETERS \\ OF PATIENTS WITH CHRONIC RENAL INSUFFICIENCY IN THE \\ START OF HEMODIALYTIC RENAL THERAPY
}

\begin{abstract}
Maíra Kufner Saloio ${ }^{1}$, Larissa De Fátima D’Amico ${ }^{2}$, Rafael Rauber ${ }^{3}$, Vanessa Uscocovich ${ }^{4}$
${ }^{1}$ Acadêmica do curso de Medicina do Centro Universitário Fundação Assis Gurgacz. ${ }^{2}$ Nutricionista pela UNICENTRO, Nutricionista Clínica Funcional com ênfase em Nefrologia e em refeições coletivas e transportadas. ${ }^{3}$ Professor colaborador, docente da cadeira de Genética do curso de Medicina FAG,

Mestre em Genética e Biologia Molecular e Doutor em Ciências: Biologia Celular e Molecular. ${ }^{4}$

Professora Orientadora, docente da cadeira de Nefrologia do Curso de Medicina FAG, Mestre em

Ensino nas Ciências da Saúde pela Faculdade Pequeno Príncipe.

* Autor Correspondente: maira saloio@hotmail.com, https://orcid.org/0000-0003-1117-6151
\end{abstract}

\section{RESUMO}

A Insuficiência Renal Crônica (IRC) é uma doença relevante na saúde pública devido sua prevalência ascendente. Ela minimiza a atividade renal, tornando os portadores dependentes de terapias de substituição (diálise), a qual simula a atividade renal para manter a homeostase hidroeletrolítica. Apesar de fundamental para a sobrevida dos portadores, ela ocasiona alterações nutricionais que devem ser monitoradas por interfirir no prognóstico e morbimortalidade. O objetivo do trabalho foi avaliar os parâmetros bioquímicos-físicos dos pacientes-dialíticos em tratamento inicial e identificar as mudanças após 3 e 6 meses associando-os a efetividade terapêutica. Trata-se de uma análise longitudinal-descritiva, tendo como base bioquímica a albumina, ferritina, hematócrito e potássio. Para base antropométrica foi feita a média das aferições da prega cutânea tricipital (PCT) e adequação ao percentil 50. Dos 22 pacientes $68,18 \%$ são homens, a idade predominante esteve entre $60-69$ anos e a doença base, a hipertensão. Com base na PCT, os pacientes estiveram em déficit grave de gordura subcutânea, enquanto que a albumina obteve melhoras gradativas, atingindo a normalidade. $O$ hematócrito reduzido associado à ferritina aumentada, confirmou anemia inflamatória, frequente nos pacientes renais crônicos. Pacientes hipercalêmicos melhoraram gradativamente. Conclui-se, que há uma real importância do fator nutricional sobre a efetividade da terapia hemodialítica.

Palavras-chave: Terapia Renal Substitutiva. Insuficiência Renal Crônica (IRC). Hemodiálise. Estado Nutricional.

ABSTRACT
Chronic Kidney Failure (CKD) is a therapeutic disease that precedes its rising
prevalence. It minimizes renal activity, making habits dependent on replacement
therapies (dialysis) a symmetrical activity for the maintenance of a hydroelectrolytic
homeostasis. Although fundamental for patients' survival, it causes nutritional changes
that must be monitored because it interferes without prognosis and morbidity and
mortality. The aim of this study was to evaluate the clinical and biochemical parameters
of dialysis patients undergoing initial treatment and appropriate to changes after 3 and
6 months, associating them with therapeutic effectiveness. This is a longitudinal and
descriptive analysis based on an albumin, ferritin, hematocrit and potassium. The 
anthropometric database was performed by the average triceps skinfold thickness (PCT) and adequacy to the 50th percentile. Of the 22 patients, $68.18 \%$ are men, predominance of age between 60-69 years and disease base, hypertension. Based on the PCT, patients apply severe subcutaneous deficit, while one band improved the gradations to normal. Reduced hematocrit associated with increased ferritin confirmed inflammatory anemia, common in chronic renal patients. Hyperkalemic patients gradually improved. It is concluded that there is a real importance of the nutritional factor on the effectiveness of hemodialysis therapy.

Key-words: Renal Replacement Therapy. Chronic Renal Insufficiency (CRF). Hemodialysis. Nutritional status.

\section{INTRODUÇÃO}

A Insuficiência Renal Crônica (IRC) é uma patologia que, de forma gradual e lenta, minimiza a atividade renal até a sua total falência. A diálise surge então como terapia para o paciente acometido pelo quadro renal e realiza de forma artificial a eliminação de substâncias orgânicas a fim de manter o equilíbrio ácido-base, eletrolítico e dos demais fatores séricos controlados pelos rins (CUPPARI, 2005).

Segundo o inquérito realizado por Sesso, et al. (2017), há contínuo crescimento tanto das taxas de incidência e prevalência em diálise, como do número absoluto de pacientes que necessitam do tratamento dialítico. Em contrapartida, a taxa de mortalidade permaneceu estável. Nesse viés, a importância está em entender um pouco mais da dinâmica físico-nutricional que o quadro patológico condiciona ao paciente renal crônico em terapia hemodialítica.

Há relevância, também, por se avaliar a questão risco-resultado ofertado pela terapia. Como todo tratamento, a diálise contém benefícios e prejuízos uma vez que abstém da regulação por feedback e é controlada por parâmetros e maquinários réplicas renais. Assim, apesar de contribuir para uma melhor qualidade de vida e prolongamento da mesma, as condições impostas pela doença somada ao tratamento dialítico resultam em variadas alterações orgânicas que favorecem a alteração nutricional dos pacientes renais crônicos (D'AMICO, FRANCO, et al., 2013).

Contudo, o presente estudo foi motivado pela necessidade de compreensão da dinâmica físico- nutricional que o quadro renal patológico condiciona tanto ao paciente doente renal agudo quanto ao paciente doente renal crônico em terapia hemodialítica, no entanto o enfoque será dado ao quadro crônico. O interesse nessa pesquisa surgiu durante atividades práticas supervisionadas na cadeira de Nefrologia durante a graduação de Medicina.

Assim, o trabalho busca compreender o fator nutricional e seus empasses em pacientes que vivenciam esse contexto hemodialítico. Assim, foi analisado como fator antropométrico a prega cutânea tricipital (PCT), a qual avalia o teor de gordura subcutânea em cada paciente. Esta, além da prega subescapular, é a mais adequada para se avaliar de forma isolada tal percentil (ACUÑA e CRUZ, 2004). Já para o viés bioquímico, a albumina foi estudada para estabelecer a relação, juntamente com a PCT do quão real seria a desnutrição, bem como para avaliar o quadro inflamatório da DRC, uma vez que os níveis de albumina se vinculam com o estado de desnutrição proteica, bem como com a inflamação intrínseca da doença e o estado volêmico do paciente (DRAIBE, KAMIMURA, et al., 2004). Hematócrito e ferritina também fizeram parte do estudo, com eles foi possível entender a presença da anemia existente na população dialisada (BUENO e FRIZZO, 2014). Para compor ainda este viés laboratorial, foi analisado o potássio sérico, importante eletrólito, que em excesso ou 
em déficit pode desencadear importantes alterações cardiológicas, sendo assim necessário seu acompanhamento, uma vez que sofre influência do fator nutricionaldietético do paciente em terapia renal de substituição (MIRA, GARAGARZA, et al., 2017).

\section{METODOLOGIA}

\subsection{TIPO DE ESTUDO}

Trata-se de um estudo analítico longitudinal e descritivo, baseado em uma pesquisa fenomenológica e descritiva, com abordagem quantitativa. Com realização de uma avaliação inicial dos parâmetros nutricionais, bioquímicos e físicos com análise da efetividade do tratamento dialítico e alterações do estado nutricional a partir da comparação desses parâmetros no tempo zero (inicio de hemodiálise), após 3 meses e 6 meses, levando em consideração a idade e o sexo dos pacientes. Foram inclusos todos os pacientes que aceitaram participar da pesquisa, tendo iniciado a hemodiálise em julho, agosto, setembro e dezembro de 2018 no centro de referência em tratamento de doenças renais, Clínica do Rim (Renalclin), a qual se encontra na macrorregião da cidade de Cascavel- PR abrangendo 25 municípios da Décima Regional de Saúde.

\subsection{AVALIAÇÃO ANTROPOMÉTRICA}

Foram realizados pinçamentos de prega cutânea tricipital direita e esquerda, sendo utilizada para a análise posterior apenas a PCT direita, devido ao grande número de pacientes com fístula dialítica em braço esquerdo. Para sua realização foi utilizado o adipômetro Innovare 2 cescorf e a verificação ocorria após a pesagem do paciente ao término da sessão hemodialítica.

\subsection{AVALIAÇÃO BIOQUÍMICA}

Foram selecionados para analise os valores séricos de albumina, ferritina, hematócrito e potássio. Estes são alguns dos parâmetros utilizados pelo próprio campus da pesquisa para acompanhamento do paciente.

\subsection{ANÁLISE DE DADOS}

Os dados coletados, antropométrico e bioquímico, foram redigidos em planilhas eletrônica Excel® e posteriormente analisados de acordo com a literatura atual.

\subsection{COMITÊ DE ÉTICA}

O presente trabalho foi aprovado pelo Comitê de Ética em Pesquisa com Seres Humanos da Faculdade Assis Gurgacz (FAG) sob o parecer 2.681.757 e encontra-se de acordo com as normas regulamentadoras de pesquisa envolvendo seres humanos, conforme normativas do Sistema CEP/CONEP. A equipe deste estudo respeita os participantes da pesquisa e a confidencialidade dos dados coletados, bem como, descreve que oferecerá o suporte necessário em eventual risco. A coleta de dados foi realizada após as assinaturas da Declaração de Permissão de Utilização dos Dados pelo local e do Termo de Consentimento Livre Esclarecido pelo paciente, respeitando a resolução nํ1 196/1996 do Conselho Nacional de Saúde. 


\section{RESULTADOS E DISCUSSÃO}

Segundo a Sociedade Brasileira de Nefrologia (SBN), a Hemodiálise (HD) consiste no tratamento de doentes renais crônicos e, também, dos doentes renais agudos, fazendo a limpeza e filtração do sangue para que substâncias prejudiciais à saúde sejam eliminadas do corpo, favorecendo, assim, o controle da pressão arterial e o equilíbrio hidrossalino do sangue. O princípio de seu funcionamento compreende a captação do sangue do paciente através de um acesso vascular - cateter ou fistula arteriovenosa- com posterior impulsão por bomba para o dialisador, circuito extracorpóreo dotado de membrana artificial. O filtro de diálise comunica o sangue à solução dialítica através de uma membrana semipermeável e o processo de limpeza ocorre (SBN), retirando o excesso hidroeletrolítico do sangue durante toda a sessão da terapia.

Os pacientes que necessitam passar por hemodiálise são portadores de Doença Renal, esta quando crônica (DRC) leva a alterações do funcionamento e à minimização da filtração renal devido às lesões irreversíveis que ocorrem no parênquima do rim (JUNIOR, 2004). Esses quadros necessitam de terapias para reposição da atividade renal, além de cuidados dietéticos entre as sessões dialíticas (MARTINS e JUNIOR, 2008). Dessa forma, o controle nutricional dos pacientes em tratamento de hemodiálise é um dos focos de atenção da equipe multidisciplinar responsável, já que o quadro nutricional tende a alterar com o tempo de HD, levando, por exemplo, a redução de proteína somática apesar do aumento do consumo proteico por quilograma de peso (ALVARENGA e ANDRADE, 2017). Isso devido ao evento ser essencialmente catobólico (PASSOS e GALLON, 2015), além da perda significativa na diálise dos aminoácidos formadores de proteínas (MARTINS e JUNIOR, 2008) (MARTINS e RIELLA, 2010). A soma desses fatores leva ao declínio do estado nutricional, sendo esse, o principal fator de risco para a desnutrição energéticoproteico do grupo hemodialítico (ALVARENGA e ANDRADE, 2017).

A frequente desnutrição encontrada nos pacientes em HD decorre da grande perda de peso somada ao hipercatabolismo, ingesta alimentar deficiente, inflamação e comorbidades associadas (PASSOS e GALLON, 2015). Medidas simples, como o controle das classes de alimentos, são importantes para reduzir o quadro de desnutrição. Fazer ingesta de carboidratos e lipídios para suprir a necessidade calórica total é fundamental para que as proteínas ingeridas pelo paciente não sejam utilizadas como fonte primária de energia (MARTINS e RIELLA, 2010).

Não obstante, o Ganho de Peso Interdialítico (GPID) pode, também, ter acepção clínica negativa devido a rápida remoção de fluídos durante a sessão hemodialítica, além de insuficiência cardíaca congestiva entre outras (FERRAZ e SOUZA, 2015). O ideal para GPID gira em torno de 3 a $5 \%$ do peso seco do paciente, ou seja, um paciente de $70 \mathrm{Kg}$ terá um GPID de 2,1 kg (3\%). É importante não passar de $2 \mathrm{Kg}$ entre as sessões e $3 \mathrm{Kg}$ nos finais de semana, para se evitar as consequências do acúmulo de líquido como, por exemplo, aumento da pressão arterial, falta de ar e edema agudo de pulmão (MARTINS e JUNIOR, 2008). E também para evitar aquelas que podem ocorrer em decorrência da grande remoção de líquido durante a HD, como cefaleia, cãibras musculares, e o queda da pressão arterial (SMITH, COSTON, et al., 2010; NERBASS, MORAIS, et al., 2011; SMITH, COSTON, et al., 2010).

A albumina tem mostrado ser o parâmetro mais eficaz para estabelecer o estado nutricional dos pacientes em terapia de HD, sendo extremamente útil na clínica para tal analise (DRAIBE, KAMIMURA, et al., 2004) (NATIONAL KIDNEY FOUNDATION, 2000). A síntese hepática de albumina sofre redução de 50\% nas primeiras 24 horas 
de ingesta insuficiente, persistindo na queda caso ocorra o prolongamento da dieta errônea (DRAIBE, KAMIMURA, et al., 2004). São vários os fatores que podem levar a hipoalbuminemia grave e, independente do fator causal, a significativa redução da albumina sérica é um veemente preditor de óbito para os pacientes com IR (GOLDSTEIN, 1998), (LOWRIE e LEW, 1990) (ISEKI, KAWAZOE e FUKIYAMA, 1993). Com isso, o objetivo clínico é afastar o paciente dos níveis séricos que elevam a mortalidade, deixando-os em um patamar que possibilite um bom prognóstico, assim, deve-se tentar manter a albumina sérica igual ou maior que $4.0 \mathrm{~g} / \mathrm{dl}$ (NATIONAL KIDNEY FOUNDATION, 2000).

Um problema que contribui para a piora nutricional em pacientes em terapia de diálise é a perda de apetite, a qual evolui com a doença e leva a anorexia. Nesses casos é necessária a administração de estimuladores de apetite para que se consiga manter a dieta e assim os níveis séricos de albumina (MARTINS e RIELLA, 2010).

Outro marcador importante da atividade renal é o potássio, que possui ação fundamental na manutenção do potencial de membrana celular necessário para o bom funcionamento de células musculares e nervosas. Esse íon tem $90 \%$ de sua eliminação realizada pelos rins e o restante pelas fezes, a inatividade renal leva, então, ao quadro de hipercalemia, também chamado de hiperpotassemia. Assim, é recomendada a avaliação do potássio plasmático periodicamente nesses pacientes para tentar mantê-los em níveis normais (3,5 a $5 \mathrm{meq} / \mathrm{l})$ evitando tanto o seu excesso como a sua falta, afim de se evitar quadros de arritmia cardíaca e morte súbita (GONÇALVEZ, 2015).

Com frequência, os pacientes renais crônicos em terapia hemodialítica desenvolvem anemia (BUENO e FRIZZO, 2014), em geral normocrômica e normocítica. É considerada grave quando hematócrito for inferior à $25 \%$, devendo antes mesmo de se iniciar a terapia, investigar o metabolismo de ferro do paciente através da ferritina (MARTINS, 2013). Esta, também analisada nos pacientes em tratamento de hemodiálise, serve como indicador de reserva de ferro, importante para se avaliar a presença de anemia quando abaixo do valor de referência ou possível infecção e inflamação quando acima dos valores normais (DIALISE, 2017).

As preocupações dietéticas se atem para tentar impedir a piora do prognóstico, uma vez que um paciente desnutrido ou com muito ganho de peso entre as diálises pode aumentar a chance de morbimortalidade, além da piora da qualidade de vida do mesmo (FERRAZ e SOUZA, 2015) (ALVARENGA e ANDRADE, 2017). As orientações individuais e específicas para cada paciente junto com o aconselhamento nutricional para modificar a alimentação e auxiliar no prognóstico, tem importância tão relevante que se tornou a conduta primeira diante de quadro renal crônico dialítico (MARTINS e RIELLA, 2010).

Apesar de há décadas se ter o conhecimento sobre a importância da terapia nutricional - com comprovação em estudos sobre a melhora na condição proteica (albumina) e manutenção do peso corporal (MARTINS e RIELLA, 2010) - só recentemente que o acompanhamento dietético ganhou mais efetividade. Visto que a intervenção nutricional não apenas consegue nortear todo o prognóstico do paciente e aliviar sintomatologias urêmicas e de irregularidades hidroeletrolíticas, bem como regular doenças correlatas que possam surgir em decorrência do quadro renal (CUPPARI, 2005).

\subsection{ALBUMINA}

O plasma sanguíneo é constituído por uma ampla variedade de proteínas em diferentes proporções, no entanto, o maior percentual proteico é estabelecido pela 
albumina (GUIZADO, 2008), totalizando um percentil aproximado de $52 \%$ do total proteico (BURNOUF, 2007).

Os hepatócitos são os responsáveis por sua síntese diária e constante a uma taxa, em indivíduo normal, de 100 a 200 mg/kg/dia (OLIVEIRA, 2016) (FILHO, ALMEIDA, et al., 2010) assim um indivíduo saudável de $70 \mathrm{~kg}$ possui produção diária de 10 a $18 \mathrm{~g}$. O fígado é o único órgão com capacidade de sintetizar a albumina e reserva 12 a $20 \%$ da sua atividade para tal finalidade, consumindo, assim, $6 \%$ da ingesta diária de nitrogênio (DRAIBE, KAMIMURA, et al., 2004). No entanto essa taxa sofre influência do estado nutricional, bem como do conteúdo coloidal hepático (FILHO, ALMEIDA, et al., 2010). A cadeia de produção ocorre inicialmente com a síntese de preproalbumina, com a retirada do peptídeo sinal forma-se a proalbumina, esta é novamente modificada com a subtração de 6 propeptideos formando, então, a albumina (SANTOS, 2010), a qual é lançada na corrente sanguínea com meia vida de, aproximadamente, 19 dias (GUIZADO, 2008), (SANTOS, 2010).

No plasma sanguíneo, a albumina é distribuída pelo corpo: 30 a 40\% segue para o espaço intravascular, enquanto que o espaço extravascular retém 60 a $70 \%$ do total liberado (OLIVEIRA, 2016). Considerando o espaço extravascular, a musculatura capta cerca de $15 \%$ do pool albumínico; a pele, apesar de representar $6 \%$ do peso corporal, adquire algo em torno de 11 a 18\% desse pool; as vísceras apresentam valores irrisórios, sendo que o próprio local da síntese [o fígado] possui menos de 1\% de albumina (ROTHSCHILD, ORATZ e SCHREIBER, 1972). Contribui em 80\% para a manutenção da pressão osmótica devido a sua proporção plasmática (SANTOS, 2010) e sua carga negativa, a qual possibilita atrair cátions, principalmente, íons sódio para o compartimento vascular (efeito Gibbs-Donnan) (KOEPPEN e STANTON, 2018) e estes, por consequência, reterem água (NGUYEN, REYES, et al., 2006).

A redução plasmática de albumina sérica pode estar associada à doença hepática, uma vez que este é o local de sua síntese; doença renal, devido eliminação via urinária, bem como com a desnutrição ou baixa ingesta proteica (GUIZADO, 2008). Essa baixa ingesta proporciona a redução da síntese de albumina pela metade logo nas primeiras 24 horas, sendo a proteína com origem hepática que mais sofre com tal processo (DRAIBE, KAMIMURA, et al., 2004) (ROTHSCHILD, ORATZ e SCHREIBER, 1972). A explicação para isso se encontra no substrato usado para cada espécie proteica: a síntese albumínica se vale de aminoácidos oriundos da quebra de proteínas hepáticas, já as demais proteínas com origem hepática possuem como substrato os aminoácidos provenientes da proteólise da musculatura esquelética (DRAIBE, KAMIMURA, et al., 2004). A idade é outro fator que favorece a redução do percentil albumínico, estima-se uma queda de $20 \%$ do total a partir dos 70 anos de idade (DRAIBE, KAMIMURA, et al., 2004). Estudos confirmaram que a hipoalbuminemia é fator preditor independente de mortalidade em pacientes submetidos à terapia dialítica (CLARO, STINGHEN e FILHO).

A albumina sérica possui capacidade de se ligar a diversas moléculas e distribuílas pelo corpo, conforme a necessidade, assim facilita a ação de moléculas insolúveis em água para que atuem em locais distantes (CURRY, BRICK e FRANKS, 1999) (GOODSELL, 2003), como os ácidos graxos. Com estes, por exemplo, ela consegue carrear até 7 moléculas ao mesmo tempo (GUIZADO, 2008). Inúmeros fármacos, também, são fortemente influenciados em sua farmacocinética com a ação da albumina, que pode aumentar ou diminuir a sua liberação dependendo da intensidade de ligação entre os compostos (WHALEN, FINKEL e PANAVELIL, 2016). Os fármacos podem ligar-se a outras proteínas plasmáticas também, mas a ligação a principal ligação se dá entre fármaco-albumina devido a maior quantidade desta na 
composição sanguínea (SHARGEL, WU-PONG e B.C.YU, 2005). A ligação da albumina às diversas moléculas garante a estas uma menor taxa de depuração e, consequentemente, meia-vida maior (NGUYEN, REYES, et al., 2006) (WHALEN, FINKEL e PANAVELIL, 2016).

Ademais, possui imensa variedade de ação fisiológica: manutenção da pressão osmótica e do pH sanguíneo; transporte, distribuição e excreção de moléculas (SANTOS, 2010). Possui certa atividade anti-inflamatória uma vez que a soro albumina se liga e remove neutrófilos com origem em oxigênio reativo, dessa forma regula a sinalização celular que ocorre no processo inflamatório $(\mathrm{KOUOH}$, GRESSIER, et al., 1999).

\subsection{FERRITINA}

Molécula descoberta na década de 30, pelo francês Laufberger (WANG, KNOVICH, et al., 2010), possui síntese hepática (UNIMED, 2016) e carga negativa conferindo, assim, a capacidade de atrair íons de ferro (Fe2+) para o seu interior, local com enzimas chamadas ferroxidase, a qual faz a conversão do íon ferroso para o férrico (Fe3+) para que o ferro permaneça ligado à ferritina (WATT, HILTON e GRAFF, 2010). Quando não ligada ao ferro, a ferritina é chamada de apoferritina (BRINGHENTI, 2011). O processo de secreção desta molécula não se encontra ainda bem estabelecido, mas já fora identificada a secreção em hepatócitos, células de Kupffer e macrófagos (WANG, KNOVICH, et al., 2010).

A quantidade total de ferro no organismo é em torno de $50 \mathrm{mg} / \mathrm{Kg}$ de peso no homem adulto e de $35 \mathrm{mg} / \mathrm{Kg}$ na mulher adulta, aproximadamente $30 \%$ dessa quantidade fica armazenada na forma de ferritina [e de hemossiderina], apenas 0,1\% desse ferro é encontrado no plasma (GODOY, TAKAKURA, et al., 2007) e a maior parte de ferro encontra-se na hemoglobina (PAIVA, RONDÓ e SHINOHARA, 200). É uma proteína armazenadora de ferro [forma solúvel de armazenamento] (GROTTO, 2008) e seus níveis séricos correspondem à realidade total desse elemento no organismo (LADERO, ALONSO, et al., 2009). Dessa forma, a sobrecarga de ferro pode ser identificada pelo aumento na ferritina sérica. Esse aumento pode ocorrer devido ao excesso real do mineral no corpo [10\% dos casos] ou por processos patológicos [maioria dos casos] (UNIMED, 2016), como por exemplo, em casos de inflamações, doenças hepáticas, estimulação imunológica, doença aguda, doença metabólica, entre outras (LADERO, ALONSO, et al., 2009).

A ferritina é outro marcador [além da hemoglobina] que diminui no paciente anêmico (D'AMICO, FRANCO, et al., 2013). É considerado o marcador sanguíneo de maior sensibilidade e especificidade para o diagnóstico da deficiência de ferro. Além disso, a redução da ferritina corresponde ao primeiro estágio da instalação da anemia, dessa forma possibilita detecção precoce da deficiência férrica (FARIA, PEREIRA, et al., 2017).

A ferritina é encontrada em maior proporção no citosol e também nas mitocôndrias, garantindo o armazenamento do ferro em sua forma solúvel e não toxica (BRINGHENTI, 2011) (HALLIWELL e GUTTERIDGE, 2007) ao impedir a agregação se seus átomos e consequente precipitação destes agregados tóxicos (GUYTON e HALL, 2006). Quando há excesso de ferro no organismo, ocorre saturação de ferritina, assim o armazenamento passa a ser de forma insolúvel através da hemossiderina, a qual representa a forma degradada da ferritina, permitindo a agregação férrica e precipitação dos tecidos (GUYTON e HALL, 2006).

Para a quantificar o ferro total é válida a relação $1 \mu \mathrm{g} / \mathrm{L}$ de ferritina sérica correspondente para cada 8-10 mg de ferro em estoque em indivíduo adulto (COOK, 
1982). A forma circulante da ferritina varia de 15 a $300 \mu \mathrm{g} / \mathrm{L}$, sendo a janela de normalidade para os homens de 15 a $300 \mu \mathrm{g} / \mathrm{L}$, já para as mulheres em idade fértil a janela se torna um pouco mais estreita, variando de 15 a $200 \mu \mathrm{g} / \mathrm{L}$ (SILVA, 2017), após a menopausa os valores se tornam similares (GROTTO, 2010).

\subsection{HEMATÓCRITO}

O hematócrito representa a porcentagem ocupada pelos eritrócitos no volume sanguíneo total e, dessa forma, é expresso em porcentagem por volume, ou seja, mililitros de hemácias por decilitros de sangue, além de correlacionar a viscosidade sanguínea (FAILACE, 2009).

Níveis alterados de hematócrito sugerem a existência de algum problema sanguíneo, podendo indicar desidratação (SERVICES, 2018), doença cardíaca, distúrbios sanguíneos (policitemia vera) em quadros de elevação do parâmetro. Já quando ocorre a redução do mesmo, pode indicar anemias, quadro de sangramento excessivo (KASVI, 2015), deficiência de vitaminas ou minerais, ou ainda, excesso de glóbulos brancos decorrente de doença crônica, infecção ou distúrbios leucocitários (leucemia/linfoma) (MAYO, 2016).

A janela de normalidade varia de acordo com o método utilizado para a sua verificação, mas de maneira geral se estabelece a faixa de 38,3 a $48,6 \%$ para os homens, enquanto que para as mulheres a faixa fica entre 35,3 a 44,9\% (MAYO, 2016). Com base nisso, o Comitê de Insuficiência Renal Aguda da Sociedade Brasileira de Nefrologia (2007) assegura que o hematócrito seja mantido acima de $30 \%$ para otimização e tratamento do paciente.

\subsection{POTÁSSIO}

O potássio participa do ajustamento nutricional necessário nos pacientes em hemodiálise, juntamente com o fosforo, proteínas, água, sódio e carboidratos (CASAS, RODRIGUES e D'AVILA, 2015). Esse íon é adquirido pela alimentação e possui papel fundamental no funcionamento celular por atuar, por exemplo, no potencial de ação das mesmas (KRUEGER-BECK, SCHEEREN, et al., 2011).

Estima-se uma distribuição de potássio pelo corpo em torno de $55 \mathrm{mEq} / \mathrm{kg}$. Dessa forma, um indivíduo de $70 \mathrm{~kg}$ possui, aproximadamente, $3.500 \mathrm{mEq}$ de potássio, sendo pelo menos $90 \%$ intracelular e o restante extracelular. Do total extracelular apenas $2 \%$ do potássio fazem parte do fluido intersticial e do plasma (50$70 \mathrm{mEq}$ ), o restante é localizado no tecido ósseo, do qual pode ser liberado gradativamente (SEGURO, MALNIC e ZATZ, 2000).

O potássio representa o cátion de maior quantidade dentro do espaço intracelular e possui uma pequena diferença de concentração quando comparada com o extracelular que varia entre 3,5 a $5,0 \mathrm{mEq} / \mathrm{l}$. Essa diferença é garantida pelo mecanismo iônico, chamado, bomba sódio-potássio-ATPase existente na membrana celular (GOLDENSTEIN e COELHO, 2009), a qual realiza o transporte ativo do potássio para dentro da célula e do sódio pra fora desta a uma proporção de $3: 3 \mathrm{Na} / \mathrm{K}$ (RIELLA, PACHALY, et al., 2010). Esse controle inter-compartimentos deve ser eficaz e rigoroso, uma vez que um pequeno desbalanço pode resultar em concentração a nível plasmático (hipercalemia e hipocalemia) prejudicial e em alguns casos fatais (GOLDENSTEIN e COELHO, 2009).

O rim é o principal representante da excreção do íon potássio com, aproximadamente, $90 \%$ o restante ocorre pelo suor e pelas fezes. A excreção via fecal ganha importância em situações de comprometimento da taxa de filtração glomerular (BLACK, 1972). É necessário maior controle na ingesta desse eletrólito nas fases 
finais da IRC devido a menor capacidade de sua excreção pelos néfrons (GONÇALVEZ, 2015).

Alguns fatores que favorecem a hipercalemia devido à maior movimentação do espaço intracelular (LIC) para o extracelular (LEC): acidose metabólica; lise celular; deficiência insulínica, deficiência de aldosterona (ROCHA, 2009) (RIELLA, PACHALY, et al., 2010). O quadro de hipercalemia pode estar isento de manifestações clínicas, no entanto, quando presentes são intensificadas na ocorrência concomitante de acidose metabólica, hiponatremia ou hipocalcemia (RIELLA, PACHALY, et al., 2010). $\mathrm{Na}$ tentativa de compensação, a hipercalemia ocasiona uma maior liberação de insulina e aldosterona para aumentar a taxa de excreção renal e a entrada de potássio na célula (GRANTHAM, 1976). No entanto, a manifestação mais importante se encontra nas repercussões cardíacas. Essas são frequentes em situações com potássio sérico acima de $8 \mathrm{mEq} / \mathrm{l}$, sendo representadas com alterações no eletrocardiograma (ECG). Dessa forma, em quadros de hipercalemia o ECG deve ser solicitado para determinar com que rapidez a hipercalemia deve ser tratada (RIELLA, PACHALY, et al., 2010).

Já a hipocalemia (potássio $<3,5 \mathrm{mEq} / \mathrm{l}$ ) pode ser condicionada por alcalose metabólica, essa é a causa mais comum (NETO e NETO, 2003); aumento da excreção ou de perdas reais - gastrointestinais/renais, que ocasionam os verdadeiros déficits de potássio (NETO e NETO, 2003); aumento da movimentação do íon para o intracelular (hipocalemia de redistribuição) (GOLDENSTEIN e COELHO, 2009). Outra situação que pode ocorrer é uma redução na ingesta de potássio com ausência de compensação na via de excreção - na compensação o aumento da ingesta de potássio aumenta excreção do íon na urina -, nesse caso significa que os rins estão impedidos de conservar potássio (RIELLA, PACHALY, et al., 2010). As possíveis manifestações hipocalêmicas ocorrem, por exemplo, a nível metabólico com comprometimento da liberação de aldosterona e hormônio do crescimento; digestivo com náuseas e íleo paralítico; cardiovascular com irregularidade do ritmo, devido alteração do potencial de membrana, demonstrado no eletrocardiograma (ECG) (EKG) com alargamento do QRS, depressão do segmento ST, diminuição de onda T (GROSZEK, 2002); neuromuscular, quando depleção significativa de potássio ocorre redução da excitabilidade neuromuscular (KABADI, 2018), podendo ocasionar desde apatia até tetania (RIELLA, PACHALY, et al., 2010); manifestações renais, com ativação de mecanismos poupadores de potássio somado a redução da excreção urinária do íon, além da impossibilidade de concentrar a urina tendo como consequência poliúria (NETO e NETO, 2003).

\subsection{ANTROPOMETRIA}

A antropometria nutricional da era moderna se iniciou durante a primeira guerra mundial com o intuito de analisar a eficiência física dos soldados (ACUÑA e CRUZ, 2004). Sua eficiência é garantida por ser uma método não-invasivo, de baixo custo e de boa disponibilidade para avaliar a composição, tamanho e proporções do corpo humano. Garante amplo leque de informações que podem ser utilizados para elaboração de estudos epidemiológicos (CUPPARI, 2005).

A análise das pregas cutâneas é uma das medidas antropométricas mais utilizados devido sua maneira simples e não invasiva de precisar a gordura corporal, bem como de poder classificar a distribuição subcutânea de gordura. Emprega-se com maior confiabilidade a prega cutânea tricipital (PCT), devido sua maior representatividade de gordura subcutânea. O resultado pode ser comparado com os padrões propostos por Jelliffe, o qual se baseou em militares europeus e mulheres 
americanas de baixa renda ou, ainda, pelo padrão proposto por Frisancho, baseado em aferições de americanos brancos homens e mulheres, que participaram do National Health and Nutrition Survey (NHANES) 1971-1974 (ACUÑA e CRUZ, 2004).

\subsection{DISCUSSÃO}

Conforme mostra a tabela 1, dos 22 pacientes que aceitaram participar da pesquisa ao iniciarem a hemodiálise na Renaclin - Clínica do Rim do Oeste do Paraná - e que aceitaram participar da pesquisa, 15 corresponderam ao sexo masculino, representando $58,18 \%$ da amostra enquanto que os 7 restantes $(31,81 \%)$ se refere ao sexo feminino.

Considerando a idade de acometimento, o maior percentual foi na faixa de 60 a 69 anos totalizando 9 dos pacientes da amostra, em segundo lugar a faixa etária mais acometida foi de 40 a 49 anos com 6 pacientes. Apenas 2 pacientes acometidos pela doença estavam com idade entre 70 a 79 anos. Já a faixa de 20 a 29 anos e 30 a 39 anos tiveram o mesmo percentil de pacientes, com apenas 1 paciente em cada uma delas.

Tabela 1. Demografia de pacientes submetidos à hemodiálise regular em uma clínica de Cascavel, 2018-2019.

\begin{tabular}{lll}
\hline & Variáveis & $\%$ \\
\hline Sexo & Masculino & $58,18 \%$ \\
& Feminino & $31,81 \%$ \\
& & \\
& $70-79$ anos & $9,09 \%$ \\
$60-69$ anos & $40,9 \%$ \\
$50-59$ anos & $13,63 \%$ \\
$40-49$ anos & $27,27 \%$ \\
$30-39$ anos & $4,54 \%$ \\
Doença Base & HAS anos & $4,54 \%$ \\
& DM & $90,9 \%$ \\
& HAS + DM & $31,81 \%$ \\
& GN & $31,81 \%$ \\
& Outros & $0,09 \%$ \\
\hline
\end{tabular}

HAS: Hipertensão arterial sistêmica; DM: diabetes mellitus; GN: glomerulopatias.

Ao analisar as doenças de base para o acometimento do paciente renal crônico, quase a totalidade dos participantes eram hipertensos representando 90,9\%; outras comorbidades presentes foram diabetes mellitus com 31,81\%, mesma porcentagem para a associação diabetes mellitus e hipertensão - todos os diabéticos do estudo são hipertensos. Glomerulonefrite acometeu $0,09 \%$ dos participantes, enquanto que outras causas foram responsáveis a 4,54\%. Este fato discorda dos estudos de MURUSSI et al (2003) que possui a nefropatia diabética como o principal fator responsável para se iniciar a terapia hemodialítica. No entanto concorda com o Inquérito Brasileiro de Diálise Crônica 2016 (2017) que possui a nefropatia hipertensiva como causa principal da DRC seguida pela nefropatia diabética. Além disso, a maior proporção de hipertensão arterial sistêmica como doença base, está associada à sua alta prevalência na população (TANAKA, 2013), bem como à própria função renal de atuar no controle da pressão arterial e de sofrer lesão em capilar glomerular nos episódios de pico hipertensivo (DRACZEVSKI e TEIXEIRA, 2011). 
Os valores obtidos da aferição em triplicada das pregas cutâneas do tríceps, foi analisada de acordo com os padrões de referência de Frisancho após adequação ao percentil 50 da PCT pela fórmula estabelecida por este. Dessa forma, na primeira abordagem aos participantes, apenas um paciente foi classificado com excesso de peso se baseando apenas na análise da PCT, na segunda e terceira análise, sua porcentagem de adequação da PCT foi minimizando gradualmente, porém se mantendo já na segunda análise em déficit grave, juntamente com os demais participantes. Os demais estiveram com déficit grave de gordura subcutânea ao longo de todo o estudo.

A albumina sérica é um dos parâmetros mais utilizados como marcador nutricional, sendo que o quadro de hipoalbuminemia, condição frequente na DRC, ocorre devido vários fatores como perda para o dialisado e perdas urinárias, além da própria questão inflamatória da doença. Ao longo do presente estudo, a taxa de hipoalbuminemia foi decrescente, sendo encontrados percentis de $44,45 \%, 22,72 \%$ e $4,54 \%$ na primeira, segunda e terceira analise temporal, respectivamente, conforme tabela 2.

Tabela 2. Resultado laboratorial da Albumina sérica obtido dos prontuários médicos dos pacientes do estudo.

\begin{tabular}{lcccccc}
\hline Exame \Tempo & \multicolumn{1}{c}{ T1 } & \multicolumn{3}{c}{ T2 } & \multicolumn{3}{c}{ T3 } \\
\hline Aumentado & No & $\%$ & No & $\%$ & № & $\%$ \\
Normal & 0 & 0 & 0 & 0 & 4 & 18,18 \\
Reduzido & 12 & 54,54 & 12 & 54,54 & 11 & 50 \\
Não realizado & 10 & 44,45 & 5 & 22,72 & 1 & 4,54 \\
Transplantado & 0 & 0 & 4 & 18,18 & 1 & 4,54 \\
Óbito & 0 & 0 & 0 & 0 & 2 & 9,09 \\
\hline
\end{tabular}

T: refere ao tempo do estudo (T1: tempo zero; T2: tempo 3 meses; T3: tempo 6 meses).

A análise da albumina em faixas de depleção, segundo a tabela 3, permite definir os pacientes em quadro de depleção leve a moderada apenas, nenhum em depleção grave. Além disso a proporção para a faixa normal aumentou ao longo do estudo, sendo que alguns pacientes obtiveram albumina aumentada, como mostrado na tabela 2. Entende-se, assim, a redução da desnutrição proteica quando o paciente inicia a terapia dialítica associada ao acompanhamento laboratorial e nutricional de uma equipe multidisciplinar.

Tabela 3. Depleção de albumina considerando apenas pacientes vivos. Considerando apenas para esta tabela como espaço amostral $A=22$ - óbito - transplantados - Não realizados.

\begin{tabular}{lccc}
\multicolumn{1}{c}{ Depleção e VR } & T1 & T2 & T3 \\
\hline Normal: > 3,5 & $54,54 \%$ & $70,58 \%$ & $68,75 \%$ \\
Leve: $\mathbf{3}$ a 3,5 & $27,27 \%$ & $11,76 \%$ & $6,25 \%$ \\
Moderada: 2,4 a 2,9 & $13,63 \%$ & $17,64 \%$ & $0 \%$ \\
Grave: $<\mathbf{2 , 4}$ & $0 \%$ & $0 \%$ & $0 \%$
\end{tabular}

VR: valor de referência; T: refere ao tempo do estudo (T1: tempo zero; T2: tempo 3 meses; T3: tempo 6 meses). Adaptado: (BOTTONI, OLIVEIRA, et al., 2001).

Segundo estudos, há três processos que favorecem a atenuação da síntese de albumina em pacientes em diálise: a própria inflamação, como já citado; a ingesta insuficiente de proteínas; e a acidose metabólica (DRAIBE, KAMIMURA, et al., 2004). Fármacos utilizados, alimentação, estado volêmico também apresentam interferência 
na saturação de albumina. A correção destes pontos, auxilia na readequação proteica da albumina e, também, dos demais eletrólitos.

A análise da prega cutânea tricipital (PCT), a qual representa o depósito de gordura corporal, associada à albumina como marcado nutricional, descreve a possível realidade nutricional dos pacientes dialíticos. Estes recebem orientação para adquirir nutrição menos calórica e ingesta de proteínas mais próximas dos níveis prescritos, favorecendo, dessa forma, a depleção de gordura. Assim, resultando no déficit de gordura corporal e redução da desnutrição proteica.

$\mathrm{Na}$ primeira abordagem do estudo apenas um paciente apresentou valor de hematócrito normal (tabela 4), todos os restantes estiveram abaixo da faixa de normalidade, com variação de 18,70 a 35,41 para os homens e 19,1 a 29,2 para as mulheres. Na segunda abordagem $100 \%$ dos pacientes vivos obtiveram hematócrito abaixo da faixa de referência., o mesmo percentil vale para a terceira análise quando descontado o número de pacientes que foram a óbito e os pacientes que realizaram transplante.

Tabela 4. Resultado laboratorial de Hematócrito obtido dos prontuários médicos dos pacientes do estudo.

\begin{tabular}{lccccccc}
\hline Exames \empo & & T1 & \multicolumn{3}{c}{ T2 } & \multicolumn{3}{c}{ T3 } \\
\hline Aumentado & No & $\%$ & No & $\%$ & № & $\%$ \\
Normal & 0 & 0 & 0 & 0 & 0 & 0 \\
Reduzido & 1 & 4,54 & 0 & 0 & 0 & 0 \\
Não realizado & 21 & 95,45 & 21 & 95,45 & 17 & 77,27 \\
Transplantado & 0 & 0 & 0 & 0 & 0 & 0 \\
Óbito & 0 & 0 & 0 & 0 & 2 & 9,09 \\
& 0 & 0 & 1 & 4,54 & 3 & 13,63 \\
\hline
\end{tabular}

T: refere ao tempo do estudo (T1: tempo zero; T2: tempo 3 meses; T3: tempo 6 meses).

Em contrapartida, ao longo do estudo foi observado, na maioria dos pacientes, a ferritina aumentada, como pode ser observado na tabela 5.

Tabela 5. Resultado laboratorial de Ferritina obtido dos prontuários médicos dos pacientes do estudo.

\begin{tabular}{lcccccc}
\hline Exames $\backslash$ Tempo & \multicolumn{2}{c}{ T1 } & \multicolumn{2}{c}{ T2 } & \multicolumn{3}{c}{ T3 } \\
\hline Aumentado & No & $\%$ & No & $\%$ & № & $\%$ \\
Normal & 17 & 77,27 & 10 & 45,45 & 13 & 59,09 \\
Reduzido & 5 & 22,72 & 9 & 40,9 & 3 & 13,63 \\
Não realizado & 0 & 0 & 1 & 4,54 & 0 & 0 \\
Transplantado & 0 & 0 & 1 & 4,54 & 1 & 4,54 \\
Óbito & 0 & 0 & 0 & 0 & 2 & 9,09 \\
\hline
\end{tabular}

T: refere ao tempo do estudo (T1: tempo zero; T2: tempo 3 meses; T3: tempo 6 meses).

Tal resultado configura a chamada "anemia inflamatória" que ocorre a partir de uma doença base de caráter inflamatório e, assim, se comporta como um sinal desta doença. A DRC se caracteriza por ser uma doença inflamatória com consequente elevação de marcadores inflamatórios, entre eles, a ferritina (ABENSUR H, 2006) (BUENO e FRIZZO, 2014).

$\mathrm{Na}$ avaliação do potássio sérico, observou-se que quase $60 \%$ da amostra iniciou a dialise com hipercalemia variando entre 5,7 a 8,2 mMol/L, enquanto que apenas 9 pacientes estiveram com potássio sérico normal (tabela 6). Na segunda análise a proporção se inverteu garantindo um percentil de quase $64 \%$ dentro da faixa de 
normalidade para o eletrólito. Já na última abordagem foi encontrado uma proporção de $36,36 \%$ dentro da janela normal e 40,90\% com nível sérico aumentado.

Tabela 6. Resultado laboratorial de Potássio obtido dos prontuários médicos dos pacientes do estudo.

\begin{tabular}{lcccccc}
\hline Exames \Tempo & \multicolumn{2}{c}{ T1 } & \multicolumn{3}{c}{ T2 } & \multicolumn{3}{c}{ T3 } \\
\hline Aumentado & No & $\%$ & № & $\%$ & № & $\%$ \\
Normal & 13 & 59,09 & 7 & 31,81 & 9 & 40,90 \\
Reduzido & 9 & 40,90 & 14 & 63,63 & 8 & 36,36 \\
Não realizado & 0 & 0 & 0 & 0 & 0 & 0 \\
Transplantado & 0 & 0 & 0 & 0 & 0 & 0 \\
Óbito & 0 & 0 & 0 & 0 & 2 & 9,09 \\
\multicolumn{2}{c}{ T: refere ao tempo do estudo (T1: tempo zero; T2: tempo 3 meses; T3: tempo 6 meses). }
\end{tabular}

Segundo (MIRA, GARAGARZA, et al., 2017), o potássio sérico se eleva com o uso de anti-hipertensivos do tipo inibidor da enzima conversora de angiotensinogênio (IECA) e beta-bloqueador, bem como na presença de hipoinsulinemia. Tal fato, associado à própria disfunção renal, pode ser entendido como um dos fatores para o maior percentil de pacientes com hipercalemia no primeiro mês de diálise, uma vez que para o presente o estudo, a mostra apresentou como doença base a hipertensão e a diabetes mellitus como as principais patologias de base. As oscilações da segunda e terceira análise estão relacionadas com a própria debilidade renal associada à diálise que pode não fazer excreção adequada do potássio, bem como à dieta individual de cada paciente, a qual exerce forte interferência na concentração sérica deste eletrólito (MIRA, GARAGARZA, et al., 2017) (TELLES e BOITA, 2015).

Ao longo do estudo houveram 4 óbitos de causas variadas que não serão discutidos nesse trabalho. Além disso, 2 pacientes foram transferidos de cidade para a realização de transplante.

\section{CONSIDERAÇÕES FINAIS}

A Insuficiência Renal Crônica (IRC) é uma doença de prevalência crescente no cenário brasileiro e torna seus portadores dependentes de terapia de substituição da atividade renal, a chamada diálise, objetivando-se a homeostase corporal e redução da mortalidade. No entanto, apesar de fundamental, a diálise tem influência nos fatores nutricionais, bioquímicos e psíquicos dos pacientes.

A albumina sérica, que se vale de marcador nutricional em vários estudos, mostrou neste trabalho a redução da desnutrição proteica ao longo dos seis meses de acompanhamento do paciente que inicia a hemodiálise associada aos cuidados nutricionais e laboratoriais. Em contrapartida, o valor antropométrico da prega cutânea tricipital, baseado na adequação ao percentil 50 da PCT, bem como na distribuição em percentis da PCT segundo gênero e idade, os pacientes foram classificados como baixo peso ao longo de todo o trabalho, excetuando um dos pacientes que no início da terapia dialítica obteve classificação de sobrepeso, mas ao final se juntou aos demais em baixo peso.

Junto com a lesão renal, a anemia surge como comorbidade fragilizando ainda mais o paciente. Nos participantes da pesquisa a anemia presente era condicionada pela inflamação intrínseca da doença renal crônica, tendo como característica a redução de hematócrito coexistindo com a elevação da ferritina. Dessa forma, a 
anemia presente não condiz com a escassez de ferro sérico, podendo ser resultante da redução na produção de eritropoietina devido ao quadro patológico.

Destaca-se as oscilações séricas de potássio, este sofre influência não apenas da taxa de filtração glomerular, mas também da alimentação e dos fármacos utilizados pelo paciente. Sendo a hipertensão uma das doenças bases da DRC, os pacientes chegam à primeira consulta ao nefrologista já em uso de IECA e beta bloqueadores favorecendo a retenção de potássio, propiciando dessa forma, o seu aumento sérico.

É fundamental entender toda a dinâmica que ocorre no organismo de cada paciente durante a diálise e entre as sessões para poder elaborar um plano de ação individualizado na tentativa de reduzir as perdas proteicas, eletrolíticas e hematogênicas, minimizando, assim, a chance de ocorrer quadros de desnutrição proteica e de anemia, por exemplo. Bem como pela necessidade de reduzir o aumento de potássio sérico, por exemplos, para se evitar cardiopatias consequentes. A compreensão de acúmulo e sobrecarga de metabólitos através dos exames laboratoriais de rotina permite melhorar a elaboração de cardápios nutricionais e orientações gerais, os quais maximizam o estado global do paciente.

Conclui-se, dessa forma, que há uma real importância do fator nutricional sobre a efetividade da terapia hemodialítica. Isso fica claro ao perceber que mediações dietéticas específicas para o doente renal crônico combinadas ao arsenal medicamentoso e terapia hemodialítica, garantem maior preservação da função renal, bem como uma maior segurança para tratamentos de distúrbios decorrentes da doença renal crônica.

\section{REFERÊNCIAS}

ABENSUR H, B. M. C. M. Aspectos Atuais da Anemia na Doença Renal Crônica. Jornal Brasileiro de Nefrologia , p. 104-107, 2006.

ACUÑA, K.; CRUZ, T. Avaliação do Estado Nutricional de Adultos. Arq Bras Endocrinol Metab vol 48 no 3, p. 345-361, 2004.

ALVARENGA, L. D. A.; ANDRADE, B. D. Análise do perfil nutricional de pacientes renais crônicos em hemodiálise em relação ao tempo de tratamento. Jornal Brasileiro de Nefrologia, p. 283-286, 2017.

BLACK, D. A. K. Potassium metabolism. In: KLEEMAN'S, M. \&. Clinical Disorders of Fluid and Electrolyte Metabolism. [S.I.]: McGraw-Hill, 1972. p. 121.

BOTTONI, A. et al. Avaliação nutricional: exames laboratoriais. In: WAITZBERG, D. Nutrição oral, enteral e parenteral na prática clínica. 3.ed. ed. São Paulo: Atheneu, 2001. p. 279-94.

BRINGHENTI, C. Alterações nos níveis de ferritina e transferrina e sua relação com doença hepática. Repositorio Unesc, julho 2011. Disponivel em: <http://repositorio.unesc.net/handle/1/613>.

BUENO, C. S.; FRIZZO, M. N. Anemia na doença renal crônica em hospital da região do noroeste do estado do Rio Grande do Sul. J Bras Nefrol, p. 36(3):304-314, 2014. BURNOUF, T. Modern Plasma Fractionation. Transfusion Medicine Reviews, p. Volume 21, Issue 2, Pages 101-117, 2007.

CASAS, J.; RODRIGUES, C. I. S.; D'AVILA, R. Educação nutricional para pacientes renais crônicos em programa de hemodiálise. Nutrire; vol 40, p. 36-44, 2015. 
CLARO, M.; STINGHEN, E. M.; FILHO, P.-F. Biomarcadores de Inflamação na Doença Renal Crônica. In: e-book Biomarcadores na Nefrologia. [S.I.]: Hugo Abensur, p. 81-89.

COOK, J. D. Clinical evaluation of iron deficiency. SEMIN HEMATOL. Vol 19, Fevereiro 1982. 6-18.

CUPPARI, L. Guia de Nutrição: Nutrição Clínica no adulto. Barueri, SP: Manole, 2005.

CURRY, S.; BRICK, P.; FRANKS, N. P. Ligação doÁcido Graxo à Albumina do Soro Humano: Novas Percepções dos Estudos Cristalográficos. Biochimica et Biophysica Acta; Vol 1441, ed 2-3, p. 131-140, 1999.

D'AMICO, L. D. F. et al. Caracterização do Estado Nutricional de Pacientes com Insuficiência Renal Crônica em. uniciencias,v. 17, n. 1, p. 17-24, 2013.

DIALISE, P. Portal Dialise, 31 maio 2017. Disponivel em: $<$ https://www.portaldadialise.com/articles/avaliar-a-funcao-renal>.

DRACZEVSKI, L.; TEIXEIRA, M. L. Avaliação do perfil bioquímico e hematológico em pacientes submetidos à hemodiálise. Revista Saúde e Pesquisa. Vol 44, n1, p. 1522, 2011.

DRAIBE, S. A. et al. Albumina sérica como marcador nutricional. Revista de Nutrição, p. 17(3):339-349, 2004.

EKG, M. My EKG o site do eletrocardiograma. Disponivel em: <http://pt.myekg.com/metabolicas-drogas/hipocalemia-ecg.html>.

FAILACE, R. Hemograma: manual de interpretação 5aed. Porto Alegre: artmed, 2009. FARIA, A. C. F. et al. Avaliação da presença de anemia e de deficiência de ferritina em crianças. Revista Brasileira de Análise Clínica, p. 365-370, 2017.

FERRAZ, S. F.; SOUZA, A. T. V. D. Estado nutricional ganho de peso interdialítico. Jornal Brasileiro de Nefrologia, p. 306-314, 2015.

FILHO, W. M. B. et al. Contribuição da Farmácia na prescrição e uso racional de Albumina. Journal einsten, 1 junho 2010. 8(2 Pt 1):215-20.

GODOY, M. F. et al. Ferritina Sérica e Coronariopatia Obstrutiva: Correlação. ABC Cardiol, p. 430-433, 2007.

GOLDENSTEIN, P.; COELHO, F. MedicinaNet, 22 nov. 2009. Disponivel em: $<$ http://www.medicinanet.com.br/sobre.htm>.

GOLDSTEIN, D. Assessment of nutritional status in renal diseases. Handbook of nutrition and the kidney (3ed), p. 45, 1998.

GONÇALVEZ, V. Portalda Diálise, 30 jan. 2015. Disponivel em: $<$ https://www.portaldadialise.com/articles/o-potassio-e-a-insuficiencia-renal>.

GOODSELL, D. S. PDB - Protein Data Bank, Janeiro 2003. Disponivel em: <http://www.pianetachimica.it/mol_mese/mol_mese_2003/01_Albumina/Albumina_1_ ita.html>.

GRANTHAM, J. J. renal transport and excretion of potassium. In: RECTOR'S, B. \&. The Kidney. [S.I.]: Saunders, 1976. p. 299.

GROSZEK, B. Saúde em movimento, 23 mar. 2002. Disponivel em: $<$ http://www.saudeemmovimento.com.br/conteudos/conteudo_frame.asp?cod_noticia $=510>$. Acesso em: 04 maio 2018 .

GROTTO, H. Z. W. Metabolismo do ferro: uma revisão sobre os principais mecanismos envolvidos em sua homeostase. Revista Brasileira de hematologia e Hemoterapia. Vol 30, p. 390-397, 2008.

GROTTO, H. Z. W. Diagnóstico laboratorial da deficiência de ferro. Revista Brasileira de Hematologia e Hemoterapia. Vol. 32, p. 22- 28, 2010. 
GUIZADO, T. R. C. Estudos Computacionais da Interação de Porfirinas e seus Complexos de Ferro com Albumina Sérica Humana. Dissertação (Mestrado em Física) - PUC. Rio de Janeiro, p. 141. 2008.

GUYTON, A. C.; HALL, J. E. Tratado de fisiologia Médica. Rio de Janeiro: Elsivier, 2006.

HALLIWELL, B.; GUTTERIDGE, J. M. C. Free Radicals in Biology and Medicine. 4ee. New York: Oxford, 2007.

ISEKI, K.; KAWAZOE, N.; FUKIYAMA, K. Serum Albumin is a strong predictor of death in chronic dialysis patients. Kidney Int, p. 44(1): 115-119, 1993.

JUNIOR, J. E. R. Brazilian Journal of Nephrology. http: //www.jbn.org.br, 2004. Disponivel em: <http://www.jbn.org.br/details/1183/pt-BR/doenca-renal-cronica-definicao--epidemiologia-e-classificacao>.

KABADI, M. U. BMJ Publishing. Best Practice, junho 2018. Disponivel em: $<$ https://bestpractice.bmj.com/topics/pt-br/59>.

KASVI. Kasvi, 09 julho 2015. Disponivel em: <https://kasvi.com.br/hemograma/>.

KOEPPEN, B. M.; STANTON, B. A. Berne \& Levi: Fisiologi $7^{a}$ ed. Rio de Janeiro: Elsevier, 2018.

$\mathrm{KOUOH}, \mathrm{F}$. et al. Antioxidant properties of albumin: Effect on oxidative metabolism of. IL Farmaco, p. v. 54, p. 659, 1999.

KRUEGER-BECK, E. et al. Potencial de ação: do estímulo à adaptação neural. Fisioterapia em Movimento; Vol. 24 n.3, 24 julho/setembro 2011. 535-547.

LADERO, J. M. et al. Oscillations in serum ferritin associated with antiviral therapy in chronic hepatitis C. Revista Española de Enfermedades Digestivas. Vol 101, p. 3140, 2009.

LOWRIE, E.; LEW, N. Death risk in hemodialysis patients: the predictive value of commonly measured variables and an evaluation of death rate differences between facilities. Am J Kidney Dis, p. 15(5):458-482, 1990.

MARTINS, C. Avaliação e Diagnóstico do Estado Nutricional de Pacientes Renais. In: RIELLA, M. C.; MARTINS, C. Nutrição e o Rim. Rio de Janeiro: Guanabara Koogan, 2013. p. 104-105.

MARTINS, C. T. B.; JUNIOR, E. R. Perguntas e Respostas sobre Nutrição em Diálise. São Paulo,SP: RCN, 2008.

MARTINS, C.; RIELLA, M. Nutrição e o Rim - Terapia Nutricional do Paciente Renal Crônico e Agudo. [S.I.]: Guanabara Koogan, 2010.

MAYO, C. Teste de hematócrito: visão geral. Fundação Mayo de Educação Médica e Pesquisa, 26 maio 2016. Disponivel em: <https://www.mayoclinic.org/testsprocedures/hematocrit/about/pac-20384728>.

MIRA, A. R. et al. Manual de Nutrição e Doença Renal. Associação Portuguesa dos Nutricionistas, Porto, Março 2017. 2- 42.

MURUSSI, M. et al. Nefropatia diabética no diabete melito tipo 2: fatores de risco e prevenção. Arquivos Brasileiros de Endocrinologia e Metabologia; Vol 47, p. 207219, 2003.

NATIONAL KIDNEY FOUNDATION, K. clinical practice guidelines for nutrition in chronic renal failure. Am J Kidney, p. 18-19, 32-37, 76-85., 2000.

NERBASS, F. B. et al. Ganho de peso interdialítico em pacientes em hemodiálise. J Bras Nefrol, p. 33(3):300-305, 2011.

NETO, O. M. V.; NETO, M. M. DISTÚRBIOS DO EQUILÍBRIO HIDROELETROLÍTICO. Medicina (Ribeirão Preto.Online); Vol36, p. 325-337, 2003. 
NGUYEN, A. et al. The pharmacokinetics of an albumin-binding Fab (AB.Fab) can be modulated as a function of affinity for albumin. Protein Engineering Desing and Selection, 19 julho 2006. v. 19, n. 7,p. 291-297.

OLIVEIRA, G. C. D. PLASMA HUMANO: COMPONENTES E DERIVADOS. Repositórios Científicosde Acesso Abertode Portugal, p. http://hdl.handle.net/10400.26/13168, 2016.

PAIVA, A. A.; RONDÓ, ; SHINOHARA, E. M. G. Parâmetros para avaliação do estado nutricional de ferro. Revista de Saúde Pública. Vol 34, p. 420-426, 200.

PASSOS, C. B. D.; GALLON, C. W. Estado nutricional e o seu impacto na saúde dos pacientes submetidos à hemodiálise: revisão de literatura. Revista Brasileira de Nutricao Clínica, p. 81-90, 2015.

RIELLA, M. C. et al. Metabolismo do Potássio. In: RIELLA Princípios de Nefrologia e Distúrbios Hidroeletrolíticos. [S.I.]: Guanabara Koogan, 2010. p. 194-218.

ROCHA, P. N. Hipercalemia. Jornal Brasileiro de Nefrologia, janeiro-março 2009. Vol 31: 5.

ROTHSCHILD, M. A.; ORATZ, M.; SCHREIBER, S. S. Albumin Synthesis. N Engl J Med, p. 286(14):748-50, 1972.

SANTOS, B. F. C. D. et al. Sociedade Brasileira de Nefrologia, 2007. Disponivel em: $<$ https://arquivos.sbn.org.br/uploads/Diretrizes_Insuficiencia_Renal_Aguda.pdf $>$.

SANTOS, P. G. Estudo do mecanismo de interação de Māngiferina e Mangiferitina com Soroalbumina. Biblioteca degital de teses e dissertações, Alfenas, 30 julho 2010. Disponivel em: <https://bdtd.unifal-mg.edu.br:8443/handle/tede/719>.

SBN. Sociedade Brasileira de Nefrologia. Disponivel em: $<$ https://sbn.org.br/publico/tratatamentos/hemodialise/>.

SEGURO, A. C.; MALNIC, G.; ZATZ, R. Distúrbio do metabolismo do potássio; Cap.8. In: ZATZ, R. Fisiopatologia Renal. [S.I.]: Atheneu, 2000. p. 123-150.

SERVICES, U. S. D. O. H. \&. H. Exames medicos: Hematócrito. Medline Plus Información de salud para usted, 2 agosto 2018. Disponivel em: <https://medlineplus.gov/spanish/pruebas-de-laboratorio/hematocrito/>.

SESSO, C. et al. Inquérito Brasileiro de Diálise Crônica 2016. J Bras Nefrol 2017;39(3), p. 261-266, 2017.

SESSO, et al. Inquérito Brasileiro de Diálise Crônica 2016. Jornal Brasileiro de Nefrologia Vol 39, 2017. 261-266.

SHARGEL, L.; WU-PONG, S.; B.C.YU, A. Applied Biopharmaceutics \& pharmacokinetics. Nova York: Mc Graw Hil, Medical Pub. Division, 2005.

SILVA, G. Nível de ferritina elevado indica necessidade de mudança de hábitos. Jonal Novo Hamburgo , 15 maio 2017.

SMITH, K. et al. Patient perspectives on fluid management in chronic hemodialysis. $\mathbf{J}$ Ren nutr, p. ;20:331-41, 2010.

TANAKA, P. K. Fatores Associados à baixa prevalência de Tratamento em Terapia Renal Substitutiva para pacientes SUS na região da Baixada Santista:um estudo exploratório. 2012. São Paulo: [s.n.], 2013.

TELLES, C; BOITA, E. D. F. IMPORTÂNCIA DA TERAPIA NUTRICIONAL COM ÊNFASE NO CÁLCIO, FÓSFORO E POTÁSSIO NO TRATAMENTO DA DOENÇA RENAL CRÔNICA. PERSPECTIVA vol39, p. 143-154, 2015.

UNIMED. O que você precisa saber sobre ferritina. Unimed Vales do Taquari e Rio Pardos, 19 jul. 2016. Disponivel em: <https://www.unimedvtrp.com.br/blog/o-quevoce-precisa-saber-sobre-ferritina/>.

WANG, W. et al. Serum ferritin: Past, present and future. Biochimica et Biophysica. Vol 1800, p. 760-769, 2010. 
WATT, R. K.; HILTON, R. J.; GRAFF, D. M. Oxido-reduction is not the only mechanism. Biochimica et Biophysica Acta, p. 745-759, 2010.

WHALEN, K.; FINKEL, R.; PANAVELIL, T. A. Farmacologia Ilustrada 6a Edição. Porto Alegre: Artmed, 2016. 1-24 p. 